\title{
Crisis management during anaesthesia: laryngospasm
}

\author{
T Visvanathan, M T Kluger, R K Webb, R N Westhorpe
}

Qual Saf Health Care 2005;14:e3 (http://www.qshc.com/cgi/content/full/14/3/e3). doi: 10.1136/qshc.2002.004275

See end of article for authors' affiliations

Correspondence to:

Professor W B Runciman, President, Australian Patient Safety Foundation, GPO Box 400, Adelaide, South Australia 5001 Australia; research@apsf. net.au

Accepted 10 January 2005
Background: Laryngospasm is usually easily detected and managed, but may present atypically and/or be precipitated by factors which are not immediately recognised. If poorly managed, it has the potential to cause morbidity and mortality such as severe hypoxaemia, pulmonary aspiration, and post-obstructive pulmonary oedema.

Objectives: To examine the role of a previously described core algorithm "COVER ABCD-A SWIFT $\mathrm{CHECK}^{\prime \prime}$, supplemented by a specific sub-algorithm for laryngospasm, in the management of laryngospasm occurring in association with anaesthesia.

Methods: The potential performance of this structured approach for the relevant incidents amongst the first 4000 reported to the Australian Incident Monitoring Study (AIMS) was compared with the actual management as reported by the anaesthetists involved.

Results: There were 189 reports of laryngospasm among the first 4000 incidents reported to AIMS. These were extracted and analysed. In $77 \%$ of cases laryngospasm was clinically obvious, but $14 \%$ presented as airway obstruction, $5 \%$ as regurgitation or vomiting, and $4 \%$ as desaturation. Most were precipitated by direct airway stimulation (airway manipulation, regurgitation, vomiting, or blood or secretions in the pharynx), but patient movement, surgical stimulus, irritant volatile agents, and failure to deliver the anaesthetic were also precipitating factors. Desaturation occurred in over $60 \%$ of cases, bradycardia in $6 \%(23 \%$ in patients aged $<1$ year), pulmonary oedema in $4 \%$, and pulmonary aspiration in $3 \%$. It was considered that, correctly applied, the combined core algorithm and sub-algorithm recommended for the diagnosis and management of laryngospasm would have led to earlier recognition of the problem and/or better management in $16 \%$ of cases.

Conclusion: Laryngospasm may present atypically and, if not promptly managed effectively, may lead to morbidity and mortality. Although usually promptly recognised and appropriately managed, the use of a structured approach is recommended. If such an approach had been used in the 189 reported incidents, earlier recognition and/or better management may have occurred in $16 \%$ of cases.
$\mathrm{L}$ aryngospasm is a form of airway obstruction that is so common and distinct that most anaesthetists consider it - to be a separate entity. ${ }^{1}$ The overall incidence in a large Scandinavian study of over 130000 anaesthetics was $0.78 \%$, and the risk was greater in certain subgroups such as children with asthma or airway infections or those undergoing oesophagoscopy or hypospadias repair, and adults undergoing anal surgery. ${ }^{1}$ In recognition of the fact that laryngospasm is a distinct entity, other forms of airway obstruction have been considered elsewhere in this set of articles. ${ }^{2}$

While laryngospasm occurs relatively frequently and is nearly always easily recognised and handled, it has the potential to cause morbidity and mortality, especially if managed poorly. Laryngospasm occasionally presents atypically and may be precipitated by factors which are not immediately recognised, increasing the potential for patient harm and further complications such as pulmonary aspiration and post-obstructive pulmonary oedema. This latter complication is especially significant as it may cause serious morbidity, and the patient may require intubation, ventilation and management in an intensive care setting. ${ }^{3}$ Risk factors include difficult intubation, nasal, oral or pharyngeal surgical site; and obesity with obstructive sleep apnoea; however, it may occur unexpectedly in any patient. ${ }^{4}$

In 1993 a "core" crisis management algorithm, represented by the mnemonic COVER ABCD-A SWIFT CHECK (the AB precedes COVER for the non-intubated patient), was proposed as the basis for a systematic approach to any crisis during anaesthesia where it is not obvious what should be done, or where actions taken have failed to remedy the situation. ${ }^{5}$ This was validated against the first 2000 incidents reported to the Australian Incident Monitoring Study (AIMS). AIMS is an ongoing study which involves the voluntary anonymous reporting of any unintended incident which reduced or could have reduced the safety margin for a patient. $^{6}$

It was concluded that, if this algorithm had been correctly applied, a functional diagnosis would have been reached within 40-60 seconds in 99\% of applicable incidents, and that the learned sequence of actions recommended by the COVER portion would have led to appropriate steps being taken to handle the $60 \%$ of problems relevant to this portion of the algorithm. ${ }^{5}$ However, this study also showed that the $40 \%$ of problems represented by the remainder of the algorithm, ABCD-A SWIFT CHECK, were not always promptly diagnosed or appropriately managed. ${ }^{5-7}$ It was decided that it would be useful, for these remaining problems, to develop a set of sub-algorithms in an easy-to use crisis management manual. ${ }^{8}$ This study reports on the potential place of the AB COVER CD-A SWIFT CHECK algorithm in the diagnosis and initial management of laryngospasm, provides an outline of a specific crisis management sub-algorithm for laryngospasm during anaesthesia, and provides an indication of the potential value of using this structured approach.

\section{METHODS}

Of the first 4000 incidents reported to AIMS, those that made reference to "airway", "obstruction", or "laryngospasm" were extracted and analysed for relevance. Presenting features, the presumed precipitating cause, the type of anaesthetic technique, the time of occurrence, the degree of 


\section{LARYNGOSPASM}

\section{SIGNS $(1,2)^{*}$}

Inspiratory stridor/airway obstruction

Increased inspiratory efforts/tracheal tug

Paradoxical chest/abdominal movements

Desaturation, bradycardia, central cyanosis

\section{THINK OF $(1,2)^{*}$}

Airway irritation and/or obstruction

Blood/secretions in the airway

Regurgitation and aspiration

Excessive stimulation/"light" anaesthesia

\section{MANAGEMENT}

Cease stimulation/surgery (2)

$100 \%$ Oxygen (3)

Try gentle chin lift/jaw thrust (4)

Request immediate assistance

Deepen anaesthesia with an IV agent (5)

Visualise and clear the pharynx/airway

If you suspect aspiration $\rightarrow$ page $16^{* *}(6)$

If you suspect airway obstruction $\rightarrow$ page $14^{* *}$ (7)

Try mask CPAP/IPPV, if this is unsuccessful

Give suxamethonium unless contraindicated (8)

Give atropine unless contraindicated (9)

Again, try mask CPAP/IPPV (10)

Intubate and ventilate (11)

\section{FURTHER CARE:}

Careful postoperative review of the patient to: confirm a clear airway

exclude pulmonary aspiration (6)

exclude post obstructive pulmonary oedema (8)

explain what happened to the patient.

There is a risk of awareness:

go and see the patient in the ward

explain again, and reassure the patient.

The sub-algorithm forms a facing page of the

Crisis Management Manual21.

* Numbers in brackets refer to Notes in the right hand panel.

** Page references refer to the Crisis Management Manual21.

\section{NOTES:}

It was judged that correct use of this algorithm would have led to earlier recognition of the problem and/or better management in $16 \%$ of 189 relevant incidents reported to AIMS

(1) $77 \%$ of cases were clinically obvious, $14 \%$ presented as airway obstruction, $5 \%$ as regurgitation, $4 \%$ as desaturation.

(2) Causes and precipitating factors: Airway manipulation $-44 \%$; blood/ secretions in the airway - $12 \%$; regurgitation/vomiting - $9 \%$; surgical stimulation $-5 \%$; moving the patient - $4 \%$; irritant volatile anaesthetics $2 \%$; failure of anaesthesia delivery system $-2 \%$.

(3) $61 \%$ of reports documented desaturation.

(4) The cricothyroid muscle is the only tensor of the vocal cords. Gentle stretching of this muscle may overcome moderate laryngospasm. In applying jaw thrust, gentle pressure should be exerted on the angle of the mandible, and not on soft tissues.

(5) Try $20 \%$ of the induction dose; this may be all that is needed ( $5 \%$ of cases were managed in this way); for more details, and for advice about children see (8) and (9) below.

(6) $3 \%$ of cases were associated with aspiration.

(7) $20 \%$ of cases presenting as airway obstruction were due to laryngospasm.

(8) Suxamethonium: Delay in relieving severe laryngospasm was associated with post-obstructive pulmonary oedema in $4 \%$ of cases; $15 \%$ of cases were managed with suxamethonium without intubation.

$0.5 \mathrm{mg} / \mathrm{kg}$ IV to relieve laryngospasm (see page 13*)

$1.0-1.5 \mathrm{mg} / \mathrm{kg}$ IV for intubation.

$4.0 \mathrm{mg} / \mathrm{kg} \mathrm{IM}$ for intubation (if no IV access).

(9) Atropine: $0.01 \mathrm{mg} / \mathrm{kg}$. Bradycardia occurred in $6 \%$ of all cases and in $23 \%$ of patients less than 1 year of age.

(10) $28 \%$ of cases were managed by mask CPAP/IPPV.

(11) $43 \%$ of cases were intubated.

These notes comprise a reverse side of a page of the Crisis Management Manual21.

* Page references refer to the Crisis Management Manual21.

Figure 1 Laryngospasm.

physiological change, and the patient's ASA status, management and outcome were considered. The AB COVER CD-A SWIFT CHECK algorithm, described elsewhere in this set of articles, ${ }^{8}$ was applied to each relevant report to determine the stages at which the problem might have been diagnosed, and to confirm that activating the COVER portion would have led to appropriate initial steps being taken. As laryngospasm is not adequately dealt with by this algorithm, a specific subalgorithm for laryngospasm was developed (see fig 1) and its putative effectiveness was tested against the relevant reports. The potential value of this structured approach-that is, the application of AB COVER CD-A SWIFT CHECK to the diagnosis and initial management of this problem, and the application of the sub-algorithm for laryngospasm (fig l, left panel)—was assessed in the light of the AIMS reports by comparing its potential effectiveness for each incident with that of the actual management, as recorded in each report.

\section{RESULTS}

Of the first 4000 AIMS reports, there were 189 cases of laryngospasm; 145 (77\%) were clinically obvious and easily diagnosed. The remaining $23 \%$ presented initially to the anaesthetist concerned as non-laryngospasm airway obstruction (27 cases, $14 \%$ ), vomiting/regurgitation (nine cases, $5 \%$ ), or desaturation (seven cases, $4 \%$ ). Table 1 shows the causes attributed.

Table 2 shows the type of anaesthetic used and time of occurrence, table 3 the physiological outcomes, and table 4 the documented management.

Table 1 Precipitating causes of laryngospasm

\begin{tabular}{lcc}
\hline Cause of laryngospasm & No of incidents & $\%$ \\
\hline Airway manipulation & 83 & 44 \\
Blood/secretions in the pharynx & 22 & 12 \\
Regurgitation/vomiting & 17 & 9 \\
Surgical stimulus & 9 & 5 \\
Moving patient & 8 & 4 \\
Irritant volatile agent & 4 & 2 \\
Failure of anaesthetic delivery system & 3 & 2 \\
Unable to determine & 43 & 22 \\
Total & 189 & 100 \\
\hline
\end{tabular}


Table 2 Type of anaesthetic and time of occurrence of laryngospasm

\begin{tabular}{|c|c|c|c|c|c|}
\hline & Induction & Maintenance & Emergence & Recovery & Number \\
\hline IPPV-ETT & 3 & 0 & 64 & 8 & 75 \\
\hline IPPV-Mask & 4 & 0 & 0 & 0 & 4 \\
\hline SR-Mask & 37 & 17 & 2 & 2 & 58 \\
\hline SR-LM & 22 & 11 & 6 & 0 & 39 \\
\hline SR-ETT & 1 & 0 & 1 & 1 & 3 \\
\hline Unknown & 2 & 2 & 2 & 4 & 10 \\
\hline Number & 69 & 30 & 75 & 15 & 189 \\
\hline
\end{tabular}

IPPV, intermittent positive pressure ventilation; ETT, endotracheal tube; SR, spontaneous respiration; LM, laryngeal mask.

The most common types of surgery associated with laryngospasm were otolaryngological and oral surgery, with 20 incidents $(11 \%)$. In cases that involved intubation, laryngospasm tended to occur mainly after extubation during the emergence and recovery stages, while those that involved spontaneous respiration with a face or laryngeal mask occurred mainly during induction or maintenance of anaesthesia. In three incidents it was considered that the patient suffered greater morbidity because skilled assistance was not immediately available.

Desaturation was associated with laryngospasm in 115 cases $(61 \%)$. Post-obstructive pulmonary oedema was considered a likely consequence in five cases $(3 \%)$ and a possible consequence in two (1\%). Pulmonary aspiration occurred in six cases (3\%). Bradycardia occurred in 11 incidents $(6 \%)$ and was more common in younger patients, occurring in five of the 22 patients less than 1 year of age $(23 \%)$, three of the 40 patients aged $1-14$ years $(8 \%)$, but in only three of the 127 patients aged over 14 years $(2 \%)$.

When the AB COVER CD-A SWIFT CHECK algorithm was applied to each report, it was considered that the majority of cases of laryngospasm would have been detected at the A (Airway) stage of the $\mathrm{AB}$ preceding COVER, if not at the SCAN level (77\% were clinically obvious), then at the CHECK level. ${ }^{5}$ It was considered that the cause of the laryngospasm, if identified, would have been detected at the A stage of $\mathrm{AB}$ in $65 \%$ of cases (airway manipulation, blood or secretions in the pharynx, vomiting or aspiration), at the Vl (Ventilation) stage of COVER in 1\% (failure of delivery of the anaesthetic due to circuit leaks), at the V2 (Vaporiser) stage of COVER in $3 \%$ (irritant volatile agent or empty vaporiser), and at the SWIFT CHECK stage in 9\% (patient movement and/or surgical stimulus). In $22 \%$ of reports there was insufficient information to determine the cause of the laryngospasm. The actions recommended by the COVER portion of the algorithm ( $100 \%$ oxygen, turning off the vaporiser and, if necessary, removing the patient from the anaesthetic machine and circuit) were all considered appropriate immediate steps. It was also considered that, although the mechanism(s) responsible may differ under different circumstances, carrying out the recommendations of the laryngospasm subalgorithm outlined in fig 1 would have constituted an acceptable course of action in all cases.

Table 3 Physiological outcomes following incidents of laryngospasm

\begin{tabular}{lcr}
\hline Outcomes & No of incidents & $\%$ \\
\hline No physiological change & 12 & 6 \\
Minor physiological change & 107 & 57 \\
Major physiological change & 67 & 35 \\
Cardiac arrest (resuscitated) & 1 & 1 \\
Unknown & 2 & 1 \\
Total & 189 & 100 \\
\hline
\end{tabular}

When the potential effectiveness of the structured approach, represented by the AB COVER CD-A SWIFT CHECK algorithm and the special sub-algorithm for laryngospasm (fig 1) was compared with that of the actual management as documented in each of the 189 incident reports, it was considered that, properly applied, the structured approach would have led to a quicker and/or better resolution of the problem in 31 cases $(16 \%)$. These were mainly cases in which problems arose because of late recognition and/or intervention (desaturation (13 cases), postoperative pulmonary oedema (five cases), cardiac arrest (one case)) or in which an inappropriate action was taken (failure to clear the airway before mask ventilation (four cases), failure to deepen anaesthesia with an intravenous agent (three cases), continuing with an irritant volatile agent which had precipitated the problem (two cases), failure to get help (two cases), and attempting to intubate a patient without the use of a muscle relaxant (one case)). In the remaining $158(84 \%)$ of incidents it was considered that the outcome would have been no worse had the algorithms been used.

\section{DISCUSSION}

Laryngospasm is commonly perceived to be a significant problem by anaesthetists, with an incidence of $0.78 \%-5 \%$ depending on surgical type, patient age, pre-existing condition, and anaesthetic technique. ${ }^{1}$ Laryngospasm as a distinct entity comprised 189 (5\%) of the first 4000 incidents reported to AIMS.

In this review of AIMS reports, over three quarters of the cases were clinically obvious with nearly half being caused by airway manipulation (for example, insertion of a laryngeal mask or Guedel airway, extubation, or suctioning). However, it is important to note that a fifth $(22 \%)$ were precipitated by either blood or secretions in the airway, most commonly with ortolaryngological or oral surgery (11\%), or by vomiting/ regurgitation $(9 \%)$, and that in six incidents pulmonary aspiration occurred because intermittent positive pressure ventilation was performed before the pharynx was cleared. Thus, although most cases of laryngospasm should be diagnosed at the $\mathrm{A}$ stage of $\mathrm{AB}$ (preceding COVER in the spontaneously breathing patient), it is important to specifically consider and exclude the possibility of blood, secretions,

Table 4 Documented management of laryngospasm

\begin{tabular}{lcc}
\hline Management & No of incidents & $\%$ \\
\hline Intubation & 82 & 43 \\
Oxygen/CPAP/IPPV & 52 & 28 \\
Suxamethonium - without intubation & 29 & 15 \\
Increasing depth of anaesthesia & 10 & 5 \\
Unknown & 16 & 9 \\
Total & 189 & 100 \\
\hline
\end{tabular}

CPAP, continuous positive airway pressure; IPPV, intermittent positive pressure ventilation. 
or other material being in the pharynx before manipulating the airway or applying positive pressure ventilation.

The COVER portion of the algorithm had a relatively low yield. Nevertheless, seven cases were detected at the C2 (Colour) phase of COVER as they presented with desaturation, four were precipitated by irritant volatile agents (that is, at the V2 stage of COVER), and three were caused by failure of the anaesthesia delivery system (two due to circuit leaks and one because of an empty vaporiser - that is, at the V2 and Vl stages of COVER, respectively).

The A SWIFT CHECK portion of the algorithm was important in the 17 patients $(9 \%)$ in whom laryngospasm was precipitated by surgical stimulus or being moved. A high degree of "situation awareness" on the part of the anaesthetist facilitates the early diagnosis of laryngospasm. The common perception that laryngospasm can be precipitated when a patient who is in a light plane of anaesthesia is stimulated was borne out by the findings of this study; more than half of the cases of laryngospasm were precipitated by airway manipulation, surgical stimulus, or moving the patient. As can be seen from table 2, laryngospasm was most frequently reported at induction with face mask or laryngeal mask use, and during emergence or recovery in patients who had been intubated.

Laryngospasm can be potentially serious. In our series 35\% of patients suffered major physiological changes and there was one cardiac arrest (table 3). Approximately one third of patients graded ASA 1 and 2 suffered major physiological changes, and two thirds of those graded ASA 3 did so.

Desaturation occurred in over $60 \%$ of patients and was the most common physiological change reported, supporting the administration of $100 \%$ oxygen with continuous positive airway pressure with a tight fitting face mask, as long as the pharynx is clear of debris and airway obstruction is incomplete. Patients with laryngospasm can deteriorate rapidly and help should be sought early; three incidents were reported in which the patient suffered morbidity because of a lack of skilled assistance. It is well recognised from the AIMS database that not only does a lack of skilled anaesthetic assistance contribute to adverse events, but that inadequately trained assistants may actually make an adverse event worse.

Post-obstructive pulmonary oedema is not uncommon and was considered to be likely to have occurred in nearly $4 \%$ of the incidents in our collection of reports of laryngospasm. The development of marked negative intrathoracic pressures due to airway obstruction is believed to be the primary pathological event in the development of pulmonary oedema in this situation..$^{1-3} 10$ The problem can be minimised by early intervention to break the laryngospasm, either by increasing the depth of anaesthesia or by the use of a muscle relaxant. It is not clear how best to prevent the problem at extubation, some advocating extubation using a "no touch" technique when patients are awake ${ }^{11}$ and others extubation under deep anaesthesia (possibly after a magnesium infusion). ${ }^{12}$

If laryngospasm cannot be relieved immediately with gentle jaw thrust, propofol should be used to increase the depth of anaesthesia. ${ }^{13}$ The rapid onset and predictability of IV agents (compared with inhalational agents which rely on alveolar ventilation for delivery) make these the agents of choice to deepen anaesthesia rapidly in this context. Note that four cases of laryngospasm in this series were thought to have been precipitated by irritant volatile anaesthetic agents. The only exception to the use of IV agents in this context may be in children with no IV access and only partial obstruction of the airway. Here halothane is acceptable. ${ }^{14}$

There is now some evidence that sevoflurane may be associated with even fewer airway complications such as laryngospasm and coughing than halothane. ${ }^{15}$ If deepening
Key messages

- There were 189 reports of laryngospasm (5\%) among the first 4000 incidents reported to the Australian Incident Monitoring Study.

- In $77 \%$ laryngospasm was clinically obvious; $23 \%$ presented in other ways (airway obstruction, regurgitation and vomiting, desaturation).

- Desaturation was the most common physiological change recorded $(61 \%)$, emphasising the importance of IPPV with $100 \%$ oxygen administration via a clear pharynx.

- $35 \%$ of patients in this series had major physiological changes (more likely in the higher ASA grades), with one cardiac arrest.

- Precipitating factors included patient movement, surgical stimulus (11\%), volatile agent irritation, and failed delivery of the anaesthetic agent.

- If laryngospasm with a clear pharynx is not relieved immediately by jaw thrust, consider deepening anaesthesia using propofol where venous access is available.

- Bradycardia is a likely complication of laryngospasm and hypoxaemia in children.

- The structured approach, correctly applied, would have resulted in a quicker and/or better resolution in $31(16 \%)$ of the 189 cases in this series.

anaesthesia fails and muscle relaxation is required, suxamethonium is the current agent of choice for its rapidity of both onset and offset. The dose required can be as little as $0.1 \mathrm{mg} / \mathrm{kg} \mathrm{IV} .{ }^{16}$ If IV access is not available, then intramuscular suxamethonium in a dose $4 \mathrm{mg} / \mathrm{kg}$ can be given. ${ }^{17}{ }^{18}$ As the laryngeal muscles are more sensitive to the effects of depolarising muscle relaxants, the beneficial effects on laryngospasm come into effect well ahead of maximum twitch suppression. In exceptional circumstances, infralingual or intraosseous drug administration can be considered for paediatric patients who require suxamethonium but who do not have vascular access. ${ }^{19} 20$

Bradycardia may also complicate laryngospasm and hypoxaemia, especially in young children, and accompanied laryngospasm in one fifth of the cases under 1 year of age. The management sub-algorithm therefore recommends the concomitant administration of atropine with suxamethonium unless specifically contraindicated.

Aspiration occurred in six cases and we believe that most of these incidents could have been avoided if careful laryngoscopy had been performed before the application of positive pressure ventilation. The use of laryngoscopy is controversial but, if sufficient intravenous agent has been used and it is performed carefully, further provocation of laryngospasm should be avoided. It is important to note that $12 \%$ of the cases reported involved blood or secretions in the pharynx, $9 \%$ regurgitation or vomiting, and 3\% pulmonary aspiration.

In conclusion, laryngospasm is common during general anaesthesia and, although usually easily recognised and managed, it may be associated with considerable morbidity and even mortality. ${ }^{1-3}$ A structured approach is recommended; this is outlined in fig 1 . It was considered that, properly applied, the use of this approach would have led to earlier recognition and/or better management in approximately $16 \%$ of the cases of laryngospasm reported. All patients who have suffered laryngospasm should be assessed 
on admission to and before discharge from the recovery ward to confirm a clear airway and to exclude pulmonary aspiration and post-obstructive pulmonary oedema. Finally, it is important that a full explanation of what happened be given to the patient and the problem clearly documented in the anaesthetic record. If a particular precipitating event was significant, or a particular action was useful in resolving the crisis, this should be clearly explained and documented.

\section{ACKNOWLEDGEMENTS}

The authors would like to thank all the anaesthetists in Australia and New Zealand who contributed to the 4000 incident reports upon which this and the other 24 papers in the Crisis Management Series are based. The coordinators of the project also thank Liz Brown for preparing the draft of the original Crisis Management Manual; Loretta Smyth for typing; Monika Bullock RN for earlier coding and classifying of data; Dr Charles Bradfield for the electronic version of the algorithms; Dr Klee Benveniste for literature research; and Drs Klee Benveniste, Michal Kluger, John Williamson and Andrew Paix for editing and checking manuscripts.

\section{Authors' affiliations}

T Visvanathan, Staff Specialist, Department of Anaesthesia, The Queen Elizabeth Hospital, Woodville, South Australia, Australia

M T Kluger, Senior Staff Specialist, Department of Anaesthesiology and Perioperative Medicine, North Shore Hospital, Auckland, New Zealand R K Webb, Senior Staff Specialist; Department of Anaesthesia and Intensive Care, The Townsville Hospital, Douglas, Queensland, Australia R N Westhorpe, Deputy Director, Department of Paediatric Anaesthesia and Pain Management, Royal Children's Hospital, Parkville, Victoria, Australia

This study was coordinated by The Australian Patient Safety Foundation, GPO Box 400, Adelaide, South Australia 5001, Australia.

\section{REFERENCES}

1 Olsson GL, Hallen B. Laryngospasm during anaesthesia. A computer-aided incidence study in 136,929 patients. Acta Anaesthesiol Scand 1984;28:567-75.

2 Visvanathan T, Kluger MT, Webb RK, et al. Crisis management during anaesthesia: obstruction of the natural airway. Qual Saf Health Care 2005; 14:e2.
3 McConkey PP. Postobstructive pulmonary oedema-a case series and review. Anaesth Intensive Care 2000;28:72-6.

4 Scarbrough FE, Wittenberg JM, Smith BR, et al. Pulmonary edema following postoperative laryngospasm: case reports and review of the literature. Anesth Prog 1997;44:110-6.

5 Runciman WB, Webb RK, Klepper ID, et al. Crisis management: validation of an algorithm by analysis of 2000 incident reports. Anaesth Intensive Care 1993;21:579-92.

6 Webb RK, Currie M, Morgan CA, et al. The Australian Incident Monitoring Study: an analysis of 2000 incident reports. Anaesth Intensive Care 1993;21:520-8.

7 Webb RK, van der Walt JH, Runciman WB, et al. Which monitor? An analysis of 2000 incident reports. Anaesth Intensive Care 1993;21:529-42

8 Runciman WB, Kluger MT, Morris RW, et al. Crisis management during anaesthesia: the development of an anaesthetic crisis management manual. Qual Saf Health Care 2005; 14:e1

9 Kluger MT, Bukofzer M, Bullock M. Anaesthetic assistants: their role in the development and resolution of anaesthetic incidents. Anaesth Intensive Care 1999:27:269-74.

10 Lang SA, Duncan PG, Shephard DAE, et al. Pulmonary oedema associated with airway obstruction. Can J Anaesth 1990;37:210-8.

11 Tsui BC, Wagner A, Cave D, et al. The incidence of laryngospasm with a "no touch" extubation technique after tonsillectomy and adenoidectomy. Anesth Analg 2004;98:327-9.

12 Gulhas N, Durmus M, Demirbilek S, et al. The use of magnesium to prevent laryngospasm after tonsillectomy and adenoidectomy: a preliminary study. Paediatr Anaesth 2003;13:43-7.

13 Afshan G, Chohan U, Qamar-Ul-Hoda M, et al. Is there a role of a small dose of propofol in the treatment of laryngeal spasm? Paediatr Anaesth 2002; 12:625-8.

14 Fisher DM, Robinson S, Brett CM, et al. Comparison of enflurane, halothane, and isoflurane for diagnostic and therapeutic procedures in children with malignancies. Anesthesiology 1985;63:647-50.

15 Walker SM, Haugen RD, Richards A. A comparison of sevoflurane with halothane for paediatric day case surgery. Anaesth Intensive Care 1997;25:643-9.

16 Chung DC, Rowbottom SJ. A very small dose of suxamethonium relieves laryngospasm. Anaesthesia 1993;48:229-30.

17 Liu LM, DeCook TH, Goudsouzian NG, et al. Dose response to intramuscular succinylcholine in children. Anesthesiology 1981;55:599-602.

18 Warner DO. Intramuscular succinylcholine and laryngospasm. Anesthesiology $2001 ; 95: 1039-40$.

19 Weiss M, Gerber AC. The substitute for the intravenous route. Anesthesiology 2001;95:1040-1.

20 Seah TG, Chin NM. Severe laryngospasm without intravenous access-a case report and literature review of the non-intravenous routes of administration of suxamethonium. Singapore Med J 1998;39:328-30.

21 Australian Patient Safety Foundation. Crisis Management Manual: COVER $A B C D$ A SWIFT CHECK. Adelaide: Australian Patient Safety Foundation, 1996, 74 pp. Available at http://www.apsf.net.au/anaesthesia.htm (accessed 6 September 2004). 